\title{
Thermal equilibrium in de Sitter space
}

\author{
Ian H. Redmount \\ Institute of Astronomy, University of Cambridge, The Observatories, Madingley Road, Cambridge, CB3 OHA, England \\ Fernando Ruiz Ruiz* \\ Department of Applied Mathematics and Theoretical Physics, University of Cambridge, \\ 3 Silver Street, Cambridge, CB3 9EW, England \\ (Received 18 July 1988; revised manuscript received 13 December 1988)
}

\begin{abstract}
Thermal-equilibrium quantum states are constructed for free scalar fields in $(N+1)$-dimensional de Sitter space. The states are described by density matrices of "thermal" form, satisfying the von Neumann equation associated with the appropriate functional Schrödinger equation. These solutions exist only for fields with mass and/or curvature coupling corresponding to conformal invariance. The temperature associated with such a state obeys the classical red-shift law. States exist with any temperature value at any given time; the zero-temperature limit is the Euclidean vacuum state. The total field energy of a thermal state above that of the Euclidean vacuum is finite and positive. This excitation energy consists of one contribution which red-shifts classically, but it can also contain a contribution which grows in time as the radius of the space.
\end{abstract}

\section{INTRODUCTION}

Thermal physics plays an important role in gravitational physics, and particularly in cosmology; thermal equilibrium of matter and fields figures at some stage in most cosmological models. Thermal states for quantum systems in flat spacetime are well understood. But spacetime curvature and dynamics affect significantly the definition and nature of thermal equilibrium. Finitetemperature quantum field theory in curved but static or stationary spacetimes has been described by Dowker and Critchley, ${ }^{1}$ Gibbons and Perry, ${ }^{2}$ Dowker and Kennedy, ${ }^{3}$ Altaie and Dowker, ${ }^{4}$ and Critchley, Davies, and Kennedy. ${ }^{5}$ Thermal equilibrium and finite-temperature theory have been defined for conformally invariant fields in conformally static spacetimes by Gibbons and Perry, ${ }^{2}$ Kennedy, ${ }^{6}$ and $\mathrm{Hu}^{7,8}$ various methods for treating the approximately thermal behavior of more general fields in more general spacetimes have been developed by, e.g., Drummond, ${ }^{9} \mathrm{Hu},{ }^{8,10,11}$ Critchley, Davies and Kennedy, Semenoff and Weiss, ${ }^{12}$ Chen and $\mathrm{Hu},{ }^{13} \mathrm{Hu}$, Critchley, and Stylianopoulos, ${ }^{14}$ and Éboli, Jackiw, and $\mathrm{Pi}^{15}$ The purpose of this work is to examine in detail thermalequilibrium states of a quantized field in a curved and $d y-$ namic spacetime, treating a particular simple example: We consider here free scalar fields in de Sitter space. This is perhaps the simplest-and best studiedquantum field theory after flat-spacetime theory; it is also of interest as underlying inflationary cosmological models. ${ }^{16}$ For generality we treat de Sitter space of arbitrary dimensionality, as this is no more difficult than four dimensions.

Since thermal states are the objects of principal interest here, it is convenient to treat the field theory via covariant functional Schrödinger formalism, ${ }^{17}$ in which the quantum states of the field are represented explicitly. Pure states are described by wave functionals, which as- sign probability amplitudes to configurations of the field on hypersurfaces of constant time. The evolution of a wave functional from one such hypersurface to another is generated by a field Hamiltonian operator; the wave functional obeys a "functional Schrödinger equation." Thermal states, being mixed states, must be represented by density matrices. The matrix elements of these operators are likewise functionals of field values on constanttime hypersurfaces. Their time evolution is governed by the von Neumann equation corresponding to the functional Schrödinger equation. The Hamiltonian in these equations can be obtained from an integral over a constant-time hypersurface of the energy-density component of the canonical stress-energy tensor for the field. Consequently we term this formulation of the theory "canonical." An alternative, equivalent functionalSchrödinger formulation is also of use here. It is based on a time-dependent rescaling of the field; in terms of this conformally transformed field the theory can be cast in a form resembling flat-spacetime theory. ${ }^{18,19}$ The Hamiltonian and functional Schrödinger and von Neumann equations in this formulation, termed "conformal," are obtained by exploiting this resemblance. These formulations of the functional Schrödinger formalism are the same as those used by Éboli, Jackiw, and $\mathrm{Pi}^{15}$ to treat nonequilibrium density matrices in flat and de Sitter spacetimes.

The desired description of a thermal state of the field, then, is given by its density matrix. We take the form of a thermal density matrix in the conformal formulation to match that in flat spacetime, in accord with the appearance of the field theory itself. The canonical form of the density matrix follows via a simple transformation. The von Neumann equation determines conditions under which a density matrix can take such a form, i.e., under which a state thus described can exist, and fixes some of its properties. All the physical features of the thermal 
state can be calculated from the density matrix thus determined.

The derivation is presented as follows. Particulars of the spacetime geometry and field theory are given in Sec. II. In Sec. III we describe the functional Schrödinger formalism and the canonical and conformal Hamiltonian operators. The form of the thermal density matrix and the conditions imposed by the von Neumann equation are obtained in Sec. IV. As an example of the calculation of physical properties using the density matrix, in Sec. V we calculate the field energy of a thermal state, as represented by the gravitational Hamiltonian. Results are summarized and discussed in Sec. VI.

Units with $\hbar=c=k_{B}=1$ are used throughout this work. Our sign conventions and general notation follow those of Misner, Thorne, and Wheeler. ${ }^{20}$

\section{FUNDAMENTALS}

De Sitter space of $N+1$ dimensions is taken here as a fixed background for the field theory. Its metric may be written

$$
\begin{aligned}
d s^{2} & =-d t^{2}+a^{2} \cosh ^{2}(t / a) d \Omega_{N}^{2} \\
& =a^{2} \sec ^{2} \eta\left(-d \eta^{2}+d \Omega_{N}^{2}\right),
\end{aligned}
$$

where $a$ is a positive constant and $d \Omega_{N}^{2}$ is the line element of the unit $N$-sphere. Comoving-observer proper time $t \in(-\infty,+\infty)$ and conformal time $\eta \in(-\pi / 2,+\pi / 2)$ are related by

$$
\eta \equiv \int_{0}^{t / a} \frac{d u}{\cosh u}=\arctan [\sinh (t / a)]
$$

The curvature scalar is $R=N(N+1) / a^{2}$, a constant.

The field $\Phi$ considered here is a real scalar field of mass $m$, with no nongravitational couplings. The action for this field on the de Sitter background is

$S=-\frac{1}{2} \int d^{N+1} x \sqrt{-g}\left[g^{\mu v} \partial_{\mu} \Phi \partial_{\nu} \Phi+\left(m^{2}+\xi R\right) \Phi^{2}\right]$,

with $x, g, g^{\mu \nu}$, and $R$ taken from the geometry (2.1a), and $\xi$ constant. The corresponding classical field equation is

$$
\left[\square-\left(m^{2}+\xi R\right)\right] \Phi=0,
$$

with $\square$ the covariant d'Alembertian in de Sitter space. General solutions of this equation can be obtained in several forms by separation of variables. ${ }^{21-23}$

\section{THE FUNCTIONAL SCHRÖDINGER EQUATION}

The quantized field theory can be treated using covariant functional Schrödinger formalism. ${ }^{17}$ Pure quantum states of the field are described by wave functionals. In a "field-coordinate" representation, these assign probability amplitudes to configurations of the field on constant-time hypersurfaces. The evolution of a wave functional from one such hypersurface to another is governed by the functional Schrödinger equation

$$
\left[i \frac{\partial}{\partial \eta}-H\right] \Psi\left[\Phi\left(\Omega_{N}\right), \eta\right]=0,
$$

where the field argument is considered as a function only of the angular coordinates $\Omega_{N}$ on the constant-time hypersurface with the given $\eta$ value. This equation can be expressed as a functional differential equation once the Hamiltonian $H$ appropriate to the theory is known.

\section{A. The "canonical" Hamiltonian}

The Hamiltonian operator can be obtained from an integral of the field energy density over a constant-time hypersurface: ${ }^{17}$

$$
H(\eta)=\int d^{N} x \sqrt{-g}\left|g^{\eta \eta}\right| T_{\eta \eta}
$$

The component $T_{\eta \eta}$ is taken from the canonical stressenergy tensor

$T_{\mu \nu}=\partial_{\mu} \Phi \partial_{\nu} \Phi-\frac{1}{2} g_{\mu \nu}\left[g^{\rho \sigma} \partial_{\rho} \Phi \partial_{\sigma} \Phi+\left(m^{2}+\xi R\right) \Phi^{2}\right] ;$

we label the resulting Hamiltonian "canonical." Its form as a functional differential operator is obtained by replacing the conjugate momentum $\Pi=\partial_{\eta} \Phi$ by the functional derivative operator $i\left(g_{\eta \eta} / \sqrt{-g}\right) \delta / \delta \Phi$; this implements the appropriate commutation relation between $\Phi$ and $\Pi$ in the field-coordinate representation. ${ }^{17}$ The result is

$$
\begin{aligned}
H(\eta)=\frac{1}{2} \int d \Omega_{N}( & -A^{1-N}(\eta) \frac{\delta^{2}}{\delta \Phi^{2}} \\
& +A^{N-1}(\eta)\left[h^{i j} \partial_{i} \Phi \partial_{j} \Phi\right. \\
& \left.\left.+A^{2}(\eta)\left(m^{2}+\xi R\right) \Phi^{2}\right]\right),
\end{aligned}
$$

with $A(\eta) \equiv a \sec \eta$, and with $d \Omega_{N}$ the volume element and $h^{i j}$ the metric on the unit $N$-sphere.

Except in the minimal-coupling $(\xi=0)$ case, the canonical stress-energy tensor (3.3) differs from the gravitational stress-energy tensor obtained by variation of the action $S$ with respect to the metric. The latter-containing terms absent from (3.3), arising from the variation of the $\xi R \Phi^{2}$ term in the action ${ }^{24}$ - appears on the right-hand side of the Einstein field equations. Nonetheless, the Hamiltonian constructed from the canonical $T_{\mu \nu}$ is the correct choice for the functional Schrödinger equation. ${ }^{25}$ This may be seen by considering the propagator for that equation. The path integral

$$
G\left[\Phi_{1}, \eta_{1} ; \Phi_{0}, \eta_{0}\right]=\int e^{i S[\Phi]} \mathscr{D} \Phi
$$

is the propagator ${ }^{17,23}$ for any state from field configuration $\Phi_{0}$ on hypersurface $\eta_{0}$ to configuration $\Phi_{1}$ at $\eta_{1}$; here the action $S$ is given by the integral (2.2) over the region between those hypersurfaces, and the functional integral is over all fields in that region having the given configurations at $\eta_{0}$ and $\eta_{1}$. The functional $G$ can be evaluated explicitly; ${ }^{23}$ it satisfies Eq. (3.1) with $H$ the 
canonical Hamiltonian (3.4). In particular, since in the de Sitter background the mass and curvature-coupling terms contribute identically to the action, the evolution of any state via $G$ depends on $m, \xi$, and $R$ only in the combination $m^{2}+\xi R$ - as the canonical Hamiltonian does, but its gravitational counterpart does not.

The Hamiltonian takes a particularly convenient form in terms of normal-mode amplitudes for the field. The field on any constant-time hypersurface can be expanded in spherical harmonics in $N$ dimensions:

$$
\Phi\left(\Omega_{N}\right)=\sum_{L} y_{L} \mathcal{Y}_{L}\left(\Omega_{N}\right)
$$

where $L$ denotes the complete set of $N$ angular momentum quantum numbers. The harmonics $\mathcal{Y}_{L}$ are eigenfunctions of the Laplacian on the unit $N$-sphere, with eigenvalues $-L^{2}=-l(l+N-1)$, where $l$ is any nonnegative integer. ${ }^{26}$ For convenience we take the $\mathcal{Y}_{L}$ (hence the amplitudes $y_{L}$ ) to be real. These functions constitute a complete orthonormal set on the $N$-sphere; hence the coefficients $y_{L}$ constitute a complete set of mutually commuting observables for the theory, equivalent to the field values $\Phi\left(\Omega_{N}\right)$. With $\Phi$ given by expansion (3.6), and $\delta / \delta \Phi$ similarly transformed via the chain rule, the Hamiltonian (3.4) takes the form of a sum of independent single-mode Hamiltonians:

$$
\begin{aligned}
H(\eta) & =\sum_{L} H_{L}(\eta) \\
& =\sum_{L}\left[-\frac{1}{2} A^{1-N}(\eta) \frac{\delta^{2}}{\delta y_{L}^{2}}+\frac{1}{2} A^{N-1}(\eta) \omega_{L}^{2}(\eta) y_{L}^{2}\right],
\end{aligned}
$$

with

$$
\omega_{L}(\eta) \equiv\left[L^{2}+\left(m^{2}+\xi R\right) A^{2}(\eta)\right]^{1 / 2}
$$

a time-dependent conformal-time frequency.

\section{B. The "conformal" Hamiltonian}

An alternative definition of the Hamiltonian can also be used, based on a time-dependent rescaling of the field. ${ }^{15,18,19}$ The theory can be expressed in terms of the dimensionless "conformal" field $\Phi_{c} \equiv A^{(N-1) / 2}(\eta) \Phi$. Then in terms of the amplitudes of a harmonic expansion of this field, viz.,

$$
\Phi_{c}\left(\eta, \Omega_{N}\right)=\sum_{L} y_{c L}(\eta) \mathcal{Y}_{L}\left(\Omega_{N}\right)
$$

the action (2.2) takes the form

$$
\begin{aligned}
S=\sum_{L} \frac{1}{2} \int d \eta[ & {\left[\frac{d y_{c L}}{d \eta}\right]^{2}-\omega_{c L}^{2}(\eta) y_{c L}^{2} } \\
& \left.-\frac{N-1}{2} \frac{d}{d \eta}\left(y_{c L}^{2} \tan \eta\right)\right],
\end{aligned}
$$

with

$$
\omega_{c L}(\eta) \equiv\left[\lambda^{2}+\left[m^{2}+\xi R-\frac{N^{2}-1}{4 a^{2}}\right] A^{2}(\eta)\right]^{1 / 2}
$$

and

$$
\lambda \equiv\left[L^{2}+\left[\frac{N-1}{2}\right]^{2}\right]^{1 / 2}=l+\frac{N-1}{2} .
$$

The last term in the action (3.9a) is a surface term, the integral of a total derivative. It has no effect on the equation of motion and contributes only an overall phase to any wave functional, via the propagator $G$. It may therefore be disregarded. (This entails only a canonical transformation of the conjugate momentum operator.) The remaining terms constitute the action for a set of harmonic oscillators, with "coordinates" $y_{c L}$ and conformal-time frequencies $\omega_{c L}$. The associated "conformal" Hamiltonian is

$$
\begin{aligned}
H_{c}(\eta) & =\sum_{L} H_{c L}(\eta) \\
& =\sum_{L}\left[-\frac{1}{2} \frac{\delta^{2}}{\delta y_{c L}^{2}}+\frac{1}{2} \omega_{c L}^{2} y_{c L}^{2}\right] .
\end{aligned}
$$

The corresponding Schrödinger equation is of the form (3.1), with the time derivative $\partial / \partial \eta$ taken at fixed $\Phi_{c}$ or $y_{c L}$, rather than at fixed $\Phi$ or $y_{L}$ as in the canonical formulation of Sec. III A.

Despite differences in form, the canonical and conformal formulations of the functional Schrödinger equation are equivalent. To each canonical wave functional $\Psi\left[\left\{y_{L}\right\}, \eta\right]$ there corresponds a unique conformal wave functional $\Psi_{c}\left[\left\{y_{c L}\right\}, \eta\right]$ and vice versa. These functionals, representing the same quantum state, assign the same relative probability amplitudes to any given field configurations. They differ by the phase factor associated with the surface term in the action (3.9a), and by a normalization factor, since they are normalized with respect to integration over different field variables. Specifically, corresponding functionals are related by

$$
\begin{aligned}
\Psi\left[\left\{y_{L}\right\}, \eta\right]=\prod_{L} & {\left[A^{(N-1) / 4}(\eta)\right.} \\
& \left.\times \exp \left[-i \frac{N-1}{4} y_{c L}^{2} \tan \eta\right]\right] \\
& \times \Psi_{c}\left[\left\{y_{c L}\right\}, \eta\right],
\end{aligned}
$$

with $y_{L}=y_{c L} A^{(1-N) / 2}(\eta)$. Given this relation, $\Psi$ satisfies Eq. (3.1) with Hamiltonian (3.7a) and $\partial / \partial \eta$ at fixed $y_{L}$ if and only if $\Psi_{c}$ satisfies the same equation with Hamiltonian (3.10) and $\partial / \partial \eta$ taken at fixed $y_{c L}$.

\section{THERMAL STATES}

A thermal-equilibrium state of the field is a mixed quantum state, described by a density matrix $\rho$ or $\rho_{c}$. The density matrix must satisfy the von Neumann equation

$$
i \frac{\partial \rho}{\partial \eta}-[H, \rho]=0
$$

or its conformal counterpart, corresponding to the functional Schrödinger equation (3.1), and is normalized to 
unit trace.

The simple form of the conformal Hamiltonian (3.10) suggests that the conformal density matrix for a thermal state should be a product of thermal density matrices for each normal-mode oscillator:

$$
\begin{aligned}
\rho_{c} & =\prod_{L} \rho_{c L} \\
& =\prod_{L}\left[2 \sinh \left(\frac{\beta_{L} \omega_{c L}}{2}\right) e^{-\beta_{L} H_{c L}}\right] .
\end{aligned}
$$

Here the inverse temperature $\beta_{L}$ may be a function of time and, for generality, of the mode $L$, although in genuine thermal equilibrium it is expected to be independent of $L$. [The temperature $\beta_{L}^{-1}$, like all energies associated with $H$ or $H_{c}$, is in terms of conformal time; the corresponding physical temperature, associated with proper time, is $\left(A \beta_{L}\right)^{-1}$.] Given this ansatz, the conformal von Neumann equation reduces to $\left(\partial \rho_{c} / \partial \eta\right)_{y_{c L}}=0$, i.e.,

$$
\begin{aligned}
\sum_{L}\left\{\frac{1}{2} \operatorname{coth}\left[\frac{\beta_{L} \omega_{c L}}{2}\right) \frac{d}{d \eta}\left(\beta_{L} \omega_{c L}\right)-\frac{d \beta_{L}}{d \eta} H_{c L}\right. \\
\left.+\sum_{k=1}^{\infty} \frac{\left(-\beta_{L}\right)^{k}}{k !}\left[H_{c L}, \frac{d H_{c L}}{d \eta}\right]^{(k-1)}\right\} \rho_{c}=0,
\end{aligned}
$$

where the iterated commutator is defined via

$$
\left[H_{c L}, \frac{d H_{c L}}{d \eta}\right]^{(j)} \equiv\left[H_{c L},\left[H_{c L}, \frac{d H_{c L}}{d \eta}\right]^{(j-1)}\right]
$$

with

$$
\left[H_{c L}, \frac{d H_{c L}}{d \eta}\right]^{(0)} \equiv \frac{d H_{c L}}{d \eta}
$$

Evaluating the commutators and the sum over $k$ yields

$$
\begin{gathered}
\sum_{L}\left[\frac{1}{2} \operatorname{coth}\left[\frac{\beta_{L} \omega_{c L}}{2}\right] \frac{d}{d \eta}\left(\beta_{L} \omega_{c L}\right)+f_{L}(\eta)+g_{L}^{(+)}(\eta) y_{c L}^{2}\right. \\
\left.\quad+2 f_{L}(\eta) y_{c L} \frac{\delta}{\delta y_{c L}}+\frac{g_{L}^{(-)}(\eta)}{\omega_{c L}^{2}} \frac{\delta^{2}}{\delta y_{c L}^{2}}\right] \rho_{c}=0
\end{gathered}
$$

with

$$
\begin{aligned}
& f_{L}(\eta) \equiv-\frac{\omega_{c L}^{2}-\lambda^{2}}{4 \omega_{c L}^{2}}\left[\cosh \left(2 \beta_{L} \omega_{c L}\right)-1\right] \tan \eta \\
& \text { and } \\
& \begin{aligned}
g_{L}^{( \pm)}(\eta) \equiv-\frac{1}{4}[ & \pm 2 \frac{d \beta_{L}}{d \eta} \omega_{c L}^{2} \\
& +\frac{\omega_{c L}^{2}-\lambda^{2}}{\omega_{c L}}\left[\sinh \left(2 \beta_{L} \omega_{c L}\right)\right. \\
& \left.\left. \pm 2 \beta_{L} \omega_{c L}\right] \tan \eta\right]
\end{aligned}
\end{aligned}
$$

and

$$
\frac{d \beta_{L}}{d \eta}=0
$$

are both satisfied.

These, then, are the necessary and sufficient conditions for a de Sitter-space scalar field to possess thermalequilibrium states, as described by Eq. (4.2). Condition (4.5a) implies that such states exist only for a conformally invariant field theory ${ }^{27}$-with $m=0$ and $\xi=(N-1) / 4 N$ - or theories equivalent to it by virtue of the identity of mass and curvature-scalar terms in de Sitter space. This accords with the result of $\mathrm{Hu},{ }^{8}$ that thermal equilibrium can be maintained only for a conformally invariant field in a conformally static spacetime, the constancy of the de Sitter-space curvature scalar allowing slightly greater latitude in the choice of field here. $\mathrm{Hu}$ observed that in the absence of conformal invariance particle, hence entropy, production disrupts thermal equilibrium; here condition (4.5a) follow directly from the von Neumann equation and the thermal ansatz (4.2), without reference to any definition of particles. (The von Neumann equation incorporates the condition of constant entropy in an equilibrium state. ${ }^{15,28}$ ) Condition (4.5a) is also equivalent to the requirement that $H_{c}$ be time independent, in which case the imaginary-time integral expression for the density matrix used by Drummond ${ }^{9}$ reduces to the thermal-equilibrium form (4.2). Condition (4.5b) implies that the physical temperature $(A \beta)^{-1}$ associated with such a state varies with time as $A^{-1}$, in accord with the classical red-shift law for the temperature of relativistic particles. The temperature is not otherwise constrained; a thermal state exists for any value of the temperature at any given time.

The description of thermal states in the canonical formulation follows from the above results. The matrix elements of the density matrix $\rho$, in the basis of amplitudes $y_{L}$, follow from those of $\rho_{c}$ via a relation corresponding to Eq. (3.11). Specifically, since $\rho_{c}$ has the form (4.2), with $H_{c L}$ given by Eq. (3.10), its matrix elements are mode products of ordinary simple-harmonic-oscillator thermal-state matrix elements: ${ }^{29}$

$$
\rho_{c}\left[\left\{y_{c L}\right\},\left\{z_{c L}\right\}\right]=\prod_{L}\left[\frac{\lambda}{\pi} \tanh \left(\frac{\beta \lambda}{2}\right)\right]^{1 / 2} \exp \left[\frac{-\lambda}{2 \sinh (\beta \lambda)}\left[\left(y_{c L}^{2}+z_{c L}^{2}\right) \cosh (\beta \lambda)-2 y_{c L} z_{c L}\right]\right),
$$

with conditions (4.5) assumed, and $\beta$ now taken independent of $L$. Since any density matrix can be written $\rho(y, z)$ 
$=\sum_{i} c_{i} \psi_{i}(y) \psi_{i}^{*}(z)$, where the $c_{i}$ are $c$ numbers and the $\psi_{i}$ some basis of wave functions, it follows from relation (3.11) that the density matrix $\rho$ corresponding to this $\rho_{c}$ has matrix elements

$$
\begin{aligned}
\rho\left[\left\{y_{L}\right\},\left\{z_{L}\right\} ; \eta\right]= & \prod_{L} A^{(N-1) / 2}(\eta)\left[\frac{\lambda}{\pi} \tanh \left[\frac{\beta \lambda}{2}\right]\right]^{1 / 2} \exp \left[\frac{-\lambda A^{N-1}(\eta)}{2 \sinh (\beta \lambda)}\left[\left(y_{L}^{2}+z_{L}^{2}\right) \cosh (\beta \lambda)-2 y_{L} z_{L}\right]\right) \\
& \times \exp \left[-i \frac{N-1}{4}\left(y_{L}^{2}-z_{L}^{2}\right) A^{N-1}(\eta) \tan \eta\right]
\end{aligned}
$$

It can be seen directly that the density matrix thus defined satisfies the canonical von Neumann equation, with Hamiltonian (3.7a). The operator form of $\rho$ can be inferred from these results and the relationship between the Hamiltonians (3.7a) and (3.10):

$$
\begin{array}{rl}
\rho=\prod_{L} & 2 \sinh \left(\frac{\beta \lambda}{2}\right) A^{(N-1) / 2}(\eta) \exp \left(-i \frac{N-1}{4} A^{N-1}(\eta) \tan \eta y_{L}^{2}\right) \\
& \quad \times \exp \left(-\beta\left\{H_{L}+\frac{1}{8} A^{N-1}(\eta)\left[(N-1)^{2}-\left(N^{2}-1\right) \sec ^{2} \eta\right] y_{L}^{2}\right\}\right) \exp \left(+i \frac{N-1}{4} A^{N-1}(\eta) \tan \eta y_{L}^{2}\right),
\end{array}
$$

where $y_{L}$ here denotes the amplitude operators. This canonical density matrix for a thermal state takes the familiar flat-spacetime form, proportional to $e^{-\beta H}$, only in $(1+1)$-dimensional de Sitter space. There the canonical and conformal formulations are identical. (This accords with a result of Semenoff and Weiss: ${ }^{12}$ Assuming a density matrix of that form, they find that equilibrium requires both conformal and minimal coupling, which coincide only in $1+1$ dimensions.)

The zero-temperature, i.e., $\beta \rightarrow \infty$, limit of these thermal states is the Euclidean ${ }^{22,30}$ vacuum state. In this limit the density matrix factors into a product of wave functionals for a single, pure quantum state. Its wave functional, in the canonical formulation, is

$$
\begin{aligned}
\Psi_{0}\left[\left\{y_{L}\right\}, \eta\right]=\prod_{L} & {\left[\frac{\lambda}{\pi} A^{N-1}(\eta)\right]^{1 / 4} } \\
& \times \exp \left[-\frac{1}{2} A^{N-1}(\eta)\right. \\
& \left.\times\left(\lambda+i \frac{N-1}{2} \tan \eta\right) y_{L}^{2}\right] \\
& \times e^{-i \lambda \eta / 2},
\end{aligned}
$$

where the final phase factor is demanded by the functional Schrödinger equation. The wave functional of the Euclidean vacuum for massive de Sitter-space scalar fields has previously been calculated in the formulation used here. ${ }^{23}$ The analytic continuation of that wave functional to mass values in accord with condition (4.5a), i.e., the
Euclidean-vacuum wave functional for fields satisfying that condition, is just this $\Psi_{0}$.

\section{THERMAL ENERGY}

The field energy of a thermal state is a simple characteristic which can be calculated from the density matrices obtained above. The energy is given by the expectation value of a Hamiltonian; for this purpose it is useful to consider the "gravitational" Hamiltonian $H_{g}$. This is given by the integral (3.2), using the $\eta \eta$ component of the gravitational stress-energy tensor ${ }^{8,24}$

$$
\begin{aligned}
T_{\mu \nu}^{(g)}= & (1-2 \xi) \partial_{\mu} \Phi \partial_{\nu} \Phi+\left(2 \xi-\frac{1}{2}\right) g_{\mu \nu} g^{\rho \sigma} \partial_{\rho} \Phi \partial_{\sigma} \Phi \\
& -\frac{1}{2} g_{\mu \nu} m^{2} \Phi^{2}+2 \xi \Phi\left(g_{\mu \nu} \square \Phi-\nabla_{\mu} \partial_{v} \Phi+\frac{1}{2} G_{\mu \nu} \Phi\right)
\end{aligned}
$$

in the integrand. The result, with the same operator implementation as for the canonical Hamiltonian, is

$$
\begin{aligned}
H_{g}(\eta)=H(\eta)-\xi N \sum_{L} & {\left[\sec ^{2} \eta A^{N-1}(\eta) y_{L}^{2}\right.} \\
& \left.+i \tan \eta\left[y_{L} \frac{\delta}{\delta y_{L}}+\frac{\delta}{\delta y_{L}} y_{L}\right]\right],
\end{aligned}
$$

with $H$ as in Eq. (3.7a). The formal (conformal-time) energy expectation value in a thermal state is the trace of $H_{g} \rho$, viz.,

$$
\begin{aligned}
\left\langle H_{g}\right\rangle_{\beta}=\sum_{L} \int\{ & -\frac{1}{2} A^{1-N} \frac{\delta^{2}}{\delta y_{L}^{2}}+\frac{1}{2} A^{N-1}\left[L^{2}+A^{2} \frac{N^{2}-1}{4 a^{2}}\right] y_{L}^{2}-\xi N \sec ^{2} \eta A^{N-1} y_{L}^{2} \\
& \left.\quad-i \xi N \tan \eta\left[y_{L} \frac{\delta}{\delta y_{L}}+\frac{\delta}{\delta y_{L}} y_{L}\right] \mid \rho\left[\left\{y_{L}\right\},\left\{z_{L}\right\} ; \eta\right]\right\}\left.\right|_{z_{L}=y_{L}} \prod_{L} d y_{L} \\
= & \frac{1}{2} \sum_{L} \operatorname{coth}(\beta \lambda / 2) \mathcal{W}_{\lambda},
\end{aligned}
$$


with

$$
\begin{aligned}
\mathcal{W}_{\lambda} \equiv \lambda+ & \frac{1}{\lambda}\left[\frac{N(N-1)}{4} \sec ^{2} \eta-\frac{(N-1)^{2}}{4}\right] \\
& \times\left[1-\frac{4 N \xi}{N-1}\right] .
\end{aligned}
$$

This is the sum of the Euclidean vacuum and thermal excitation energies of the field. The series diverges for any value of the temperature. The divergences, however, are temperature independent, and are just those associated with the vacuum energy; the counterterms involved in the regularization or renormalization of $\left\langle H_{g}\right\rangle_{\beta}$ are likewise just those for the zero-temperature energy. The thermal contribution to the energy, therefore, is given by the difference between the expectation value $(5.3 \mathrm{a})$ and the $\beta \rightarrow \infty$ limit of that expression. ${ }^{1,3-9,11,13,14}$ This difference is

$$
\begin{aligned}
\left\langle H_{g}\right\rangle_{\beta}-\left\langle H_{g}\right\rangle_{\mathrm{vac}} & =\sum_{L}\left(e^{\beta \lambda}-1\right)^{-1} \mathcal{W}_{\lambda} \\
& =\sum_{l=0}^{\infty} \frac{D_{N}(l)}{e^{\beta \lambda}-1} \mathcal{W}_{\lambda},
\end{aligned}
$$

where

$$
\begin{aligned}
D_{N}(l) & =\frac{(2 l+N-1)(l+N-2) !}{(N-1) ! l !} \\
& =\frac{2 \lambda[\lambda+(N-3) / 2] !}{(N-1) ![\lambda-(N-1) / 2] !}
\end{aligned}
$$

is the degeneracy of modes with total angular momentum $l$, i.e., the number of harmonics $\mathcal{Y}_{L}$ with that totalangular-momentum quantum number. ${ }^{26}$ Owing to the "Bose-Einstein factor" in the summand, the difference (5.4a) is finite. It is positive for all positive temperatures.

The physical (proper-time) excitation energy of a thermal state above the Euclidean vacuum is $A^{-1}$ times the difference $(5.4 a)$. This may be written

$$
\begin{aligned}
E_{\beta}(t)= & \frac{1}{a \cosh (t / a)}\left[F_{N}(\beta)\right. \\
& \left.\quad-\frac{(N-1)^{2}}{4}\left(1-\frac{4 N \xi}{N-1}\right) G_{N}(\beta)\right] \\
& +\frac{\cosh (t / a)}{a} \frac{N(N-1)}{4}\left[1-\frac{4 N \xi}{N-1}\right) G_{N}(\beta),
\end{aligned}
$$

with

$$
F_{N}(\beta) \equiv \sum_{l=0}^{\infty} \frac{\lambda D_{N}(l)}{e^{\beta \lambda}-1}
$$

and

$$
G_{N}(\beta) \equiv \sum_{l=0}^{\infty} \frac{D_{N}(l)}{\lambda\left(e^{\beta \lambda}-1\right)} .
$$

The functions $F_{N}$ and $G_{N}$ are finite and positive; they depend only on the dimension and temperature, increasing monotonically with increasing temperature (decreasing $\beta)$. For example, in $(3+1)$-dimensional de Sitter space they can be cast in the forms

$$
F_{3}(\beta)=\frac{3}{8} \sum_{k=1}^{\infty} \frac{1}{\sinh ^{4}(k \beta / 2)}+G_{3}(\beta)
$$

and

$$
G_{3}(\beta)=\frac{1}{4} \sum_{k=1}^{\infty} \frac{1}{\sinh ^{2}(k \beta / 2)} .
$$

Since $\sinh x$ is greater than $x$ and less than $e^{x} / 2$ for all positive $x$, the bounds

$$
\frac{e^{\beta}+7}{e^{2 \beta}-1}<F_{3}(\beta)<\frac{\pi^{4}}{15 \beta^{4}}+\frac{\pi^{2}}{6 \beta^{2}}
$$

and

$$
\frac{1}{e^{\beta}-1}<G_{3}(\beta)<\frac{\pi^{2}}{6 \beta^{2}}
$$

on these functions follow directly. The functions can also be evaluated as sums of generalized Riemann $\zeta$ functions. $^{4}$

The thermal excitation energy $E_{\beta}$, then, consists of one contribution which obeys the classical red-shift law, varying as $A^{-1}$, and in certain cases another which varies as $A$, growing without bound at late times. (The corresponding energy density contributions are just the energy terms divided by the spatial volume $V_{N}=2 \pi^{(N+1) / 2} A^{N} / \Gamma[(N+1) / 2]$, as the thermal state is rotationally invariant, i.e., they vary as $A^{-1-N}$ and $A^{+1-N}$, respectively. In contrast, the vacuum energy density can only be a cosmological-constant contribution, the corresponding energy varying as $A^{N}$, by virtue of the de Sitter invariance of the Euclidean vacuum.) Because of the form of the gravitational Hamiltonian, $E_{\beta}$ differs for fields satisfying condition (4.5a) but with different $m$ and $\xi$ values. In the actual massless, conformally coupled case, the $G_{N}$ terms in Eq. (5.5a) vanish, and $E_{\beta}$ consists entirely of the $F_{N}$ contribution with classical time dependence. This is the result obtained, for any field satisfying condition (4.5a), if $\rho_{c}$ and the conformal Hamiltonian are used to calculate the energy; it also accords with the results of Kennedy, ${ }^{6} \mathrm{Hu},{ }^{7,8}$ and Drummond. ${ }^{9}$ For fields with nonzero mass and correspondingly smaller $\xi$ values, the $G_{N}$ terms add a positive contribution to the energy, including the component varying as $A$. This includes the minimally coupled case, with $\xi=0$ and $m^{2}=\left(N^{2}-1\right) /$ $4 a^{2}$, for which $E_{\beta}$ takes the value which would be obtained for any case if the canonical Hamiltonian were used as the measure of energy. The growing component of the excitation energy is thus a consequence of the breaking of strict conformal invariance, a form of "superadiabatic amplification." 31 Such effects were found by $\mathrm{Hu}^{8}$ and $\mathrm{Chen}$ and $\mathrm{Hu}^{13}$ for fields in quasi-equilibrium when conformal invariance was broken; here the constant curvature of de Sitter space allows thermal equilibrium to be maintained.

In all cases the field excitation energy $E_{\beta}$ neglects the gravitational self-energy of the field. The "back reac- 
tion" of the spacetime geometry to the excitation energy, via the Einstein field equations, is disregarded in fixing the geometry as de Sitter space, just as it is in flatspacetime field theory.

\section{CONCLUSIONS}

Explicit density matrices for thermal-equilibrium states, functionals of field values on constant-time hypersurfaces similar in form to such density matrices in flat spacetime, can be constructed as shown here for some de Sitter-space scalar fields. The field theory involved must either be conformally invariant, i.e., massless and conformally coupled, or equivalent to a conformally invariant theory by virtue of the identity of mass and scalarcurvature terms in de Sitter space. That is, the field must obey the same classical field equation, and have the same value of the action, as in a conformally invariant theory. This requirement, expressed by condition (4.5a), follows directly from the functional von Neumann equation for the thermal states. It is a general result that thermal equilibrium can obtain only for conformally invariant fields in conformally static spacetimes; ${ }^{8}$ the special case of de Sitter space, with its constant scalar curvature, admits fields with a range of masses and curvature couplings for which thermal equilibrium can be defined. Other Friedmann-Robertson-Walker spacetimes, with constant scalar curvature admit a similar latitude, e.g., in the radiation-dominated case, with $R=0$, massless scalar fields with any curvature coupling can have thermalequilibrium states. ${ }^{14}$

The requirement $(4.5 \mathrm{a})$ also implies that these thermal states cannot be obtained directly from the dimensional reduction of any states of a scalar field in higherdimensional Minkowksi space, in which de Sitter space can be embedded. The fields imposed on de Sitter space by even a massless field in such an embedding space all have $m^{2}+\xi R$ values larger than a bound which exceeds the value condition $(4.5 \mathrm{a})$ specifies. $^{23}$

The temperatures associated with these thermal states obey the classical red-shift relation for temperatures of relativistic particles. That is, the physical temperature of a state varies in time inversely as the radius of the space. This too is a consequence of the functional von Neumann equation, expressed by condition (4.5b); it is also a general result for scalar fields in thermal equilibrium in con- formally static spacetimes. ${ }^{8}$

Possible temperatures for thermal states range over all non-negative values, with the zero-temperature limit (at fixed time) being the Euclidean vacuum state. This association of the Euclidean vacuum with zero temperature, appropriate to a pure state, means that the temperature parametrizing these thermal states is a feature distinct from the positive Gibbons-Hawking ${ }^{32}$ temperature characterizing Euclidean-vacuum field fluctuations, or two-point functions, or "detector responses.",33

The excitation energy of a thermal state above the Euclidean vacuum follows from the expectation value of the gravitational Hamiltonian, calculated using the density matrices obtained here. The result is a mode sum of energies, weighted by factors of the familiar Bose-Einstein form. Because the form of the gravitational Hamiltonian, unlike those of the canonical and conformal Hamiltonians which govern the evolution of the states, differs for fields satisfying condition (4.5a) with different masses and curvature couplings, the excitation energy behaves differently for different such fields. For the massless, conformally coupled field, the energy red-shifts classically, varying in time inversely as the radius of the space; the result agrees with earlier calculations of thermal energies in Friedmann-Robertson-Walker spacetimes. ${ }^{6-9}$ For massive fields with other curvature couplings, including minimal coupling, the energy contains an additional contribution which grows directly as the radius of the space. This is an example of "superadiabatic amplification," 31 owing to the breaking of strict conformal invariance. The energy of near-equilibrium nonconformally invariant fields in more general spacetimes behaves similarly; $;^{8,13}$ in other Friedmann-Robertson-Walker spacetimes with constant scalar curvature (such as radiation-dominated ones), in which thermal equilibrium is possible for nonconformally coupled fields, the same effect should occur.

\section{ACKNOWLEDGMENTS}

F.R.R. is grateful to Professor Stephen Hawking and the Department of Applied Mathematics and Theoretical Physics for their hospitality in Cambridge, and to the Spanish Ministry of Education and The British Council for financial support. Support for I. H. R. was provided by the United Kingdom Science and Engineering Research Council.
*On leave of absence from the Departmento de Física Teórica, Universidad Complutense de Madrid.

${ }^{1}$ J. S. Dowker and R. Critchley, Phys. Rev. D 15, 1484 (1977).

${ }^{2}$ G. W. Gibbons and M. J. Perry, Proc. R. Soc. London A358, 467 (1978).

${ }^{3}$ J. S. Dowker and G. Kennedy, J. Phys. A 11, 895 (1978).

${ }^{4}$ M. B. Altaie and J. S. Dowker, Phys. Rev. D 18, 3557 (1978).

${ }^{5}$ R. Critchley, P. C. W. Davies, and G. Kennedy, Phys. Lett. 112B, 331 (1982).

${ }^{6}$ G. Kennedy, J. Phys. A 11, L77 (1978).

${ }^{7}$ B. L. Hu, Phys. Lett. 103B, 331 (1981).
${ }^{8}$ B. L. Hu, Phys. Lett. 108B, 19 (1982).

${ }^{9}$ I. T. Drummond, Nucl. Phys. B190, 93 (1981).

${ }^{10}$ B. L. Hu, in The Very Early Universe, edited by G. W. Gibbons, S. W. Hawking, and S. T. C. Siklos (Cambridge University Press, Cambridge, England, 1983), pp. 343-352.

${ }^{11}$ B. L. Hu, Phys. Lett. 123B, 189 (1983).

${ }^{12}$ G. Semenoff and N. Weiss, Phys. Rev. D 31, 689 (1985).

${ }^{13}$ L. F. Chen and B. L. Hu, Phys. Lett. 160B, 36 (1985).

${ }^{14}$ B. L. Hu, R. Critchley, and A. Stylianopoulos, Phys. Rev. D 35, 510 (1987).

${ }^{15}$ O. Éboli, R. Jackiw, and S.-Y. Pi, Phys. Rev. D 37, 3557 
(1988).

${ }^{16}$ A. H. Guth, Phys. Rev. D 23, 347 (1981).

${ }^{17}$ K. Freese, C. T. Hill, and M. Mueller, Nucl. Phys. B255, 693 (1985).

${ }^{18}$ B. Ratra, Phys. Rev. D 31, 1931 (1985).

${ }^{19}$ S. Wada, Phys. Rev. Lett. 59, 2375 (1987).

${ }^{20} \mathrm{C}$. W. Misner, K. S. Thorne, and J. A. Wheeler, Gravitation (Freeman, San Francisco, 1973), frontispiece.

${ }^{21}$ M. Gutzwiller, Helv. Phys. Acta 29, 313 (1956).

${ }^{22}$ N. A. Chernikov and E. A. Tagirov, Ann. Inst. Henri Poincaré 9, 109 (1968).

${ }^{23}$ I. H. Redmount and S. Takagi, Phys. Rev. D 37, 1443 (1988).

${ }^{24}$ N. D. Birrell and P. C. W. Davies, Quantum Fields in Curved Space (Cambridge University Press, Cambridge, 1982), pp. 87 and 88.

25J. Traschen and C. T. Hill, Phys. Rev. D 33, 3519 (1986).
${ }^{26}$ Higher Transcendental Functions (Bateman Manuscript Project), edited by A. Erdélyi, W. Magnus, F. Oberhettinger, and F. G. Tricomi (McGraw-Hill, New York, 1953), Vol. II, pp. 232-242.

${ }^{27}$ Birrell and Davies, Quantum Fields in Curved Space (Ref. 24), p. 44.

${ }^{28}$ H. E. Kandrup, Phys. Rev. D 37, 3505 (1988).

${ }^{29}$ R. P. Feynman, Statistical Mechanics (Benjamin, Reading, MA, 1972), p. 51.

${ }^{30}$ B. Allen, Phys. Rev. D 32, 3136 (1985).

${ }^{31}$ M. S. Turner and L. M. Widrow, Phys. Rev. D 37, 3428 (1988).

${ }^{32}$ G. W. Gibbons and S. W. Hawking, Phys. Rev. D 15, 2738 (1977).

${ }^{33}$ A. Vilenkin and L. H. Ford, Phys. Rev. D 26, 1231 (1982). 